\title{
Remanufacturability evaluation for waste mechanical product from technical point of view
}

\author{
Junli Shi ${ }^{1, a,{ }^{*}}$, Yajun Wang ${ }^{2, b}$ and Jinshi Cheng ${ }^{3, c}$ \\ 1Dalian Polytechnic University, Dalian 116034, China \\ ${ }^{2}$ Dalian University of Technology, Dalian 116024, China \\ ashijunli0124@163.com, bwangyj@dlpu.edu.cn, cchengjsh@dlpu.edu.cn \\ ${ }^{* *}$ Corresponding author:Junli Shi
}

Keywords: Remanufacturability, Mechanical product, Technical index.

Abstract. In this paper, a remanufacturability evaluation method for mechanical product from the technical point is put forward, according to the remanufacturing process, five remanufacturing technical feasibility indexes of disassembly, cleaning, testing, repairing and assembly are designed scientific and quantitatively, and the remanufacturability criteria is proposed. Finally the the technical feasibility indexes and remanufactaribility of WD615.87 diesel engine is analyzed, the results show that the remanufacturing technical feasibility is good.

\section{Introduction}

Waste mechanical product remanufacturing is an excellent production mode of resources saving, energy reduction and environmental protection ${ }^{[1-2]}$. However, for waste mechanical products, because of the differences for the original quality, working conditions, as well as damage extent, the remanufacturing possibility is different, therefore, before an old mechanical products being remanufactured, its remanufacturability from technical aspects should firstly be evaluated to ensure the remanufactured product can meet the needs of customers ${ }^{[3]}$.

\section{Remanufacturing technical feasibility index of waste mechanical product}

In this study, according to the technical process of remanufacturing, the remanufacturability of waste mechanical product would include five indexes of disassembly, cleaning, testing, repair and assembly.

\subsection{Disassembly index $\mu_{d}$}

Disassembly is the process of decomposing mechanical product into individual parts by means of tools and equipments. Generally, it is mainly a decomposition process of fittings and assembly relations. For the known types of mechanical products, the disassembly method, process and equipment would be determined, and the disassembly equipment is mainly through the manual process. Therefore, the disassembly index is expressed as:

$$
\mu_{d}=1
$$

\subsection{Cleaning index $\mu_{c}$}

Cleaning is a series processes of making the dirty surface of the waste parts to a certain degree of cleanliness, which is using mechanical, physical, chemical or electrochemical methods to remove the dirt attached to the surface of the waste parts. The commonly used cleaning methods are: blow, wipe, bake and wash. According to the difficulty of cleaning method, the priority matrix is obtained, as shown in Table 1. Scoring principles are that, one cleaning method (row) is much more difficult than another method (column), it will get 5 points, one method of cleaning (row) is more difficult than the other cleaning method (column), it will get 3 point, one cleaning method (line) is equal to another other (column), it will get 3 point, the difficulty of one cleaning method (row) is equal to the other (column), it will get 1 point, if conversely, it will take the reciprocal of the point values ${ }^{[4]}$. The relative 
importance of each cleaning method can be obtained through the point calculation, and then the cleaning scores are obtained.

Table 1 The determination of cleaning scores

\begin{tabular}{ccccccccc}
\hline $\begin{array}{c}\text { Cleaning } \\
\text { method } \\
\text { (row) }\end{array}$ & \multicolumn{3}{c}{ Cleaning method (column) } & Score & $\begin{array}{c}\text { Relative } \\
\text { importance } /(\%)\end{array}$ & $\begin{array}{c}\text { Approximate } \\
\text { cleaning score }\end{array}$ & $\begin{array}{c}\text { Cleaning } \\
\text { score }\end{array}$ \\
\cline { 2 - 5 } & Blow & Wipe & Bake & Wash & & 7 & 1.00 & 1 \\
Blow & 1.0 & 0.3 & 0.2 & 0.2 & 1.7 & 7 & 2.71 & 3 \\
Wipe & 3.0 & 1.0 & 0.3 & 0.3 & 4.6 & 18 & 5.88 & 6 \\
Bake & 5.0 & 3.0 & 1.0 & 1.0 & 10.0 & 38 & 5.88 & 6 \\
Wash & 5.0 & 3.0 & 1.0 & 1.0 & 10.0 & 38 & 15.47 & - \\
Total & & & & & 26.3 & 100 & & \\
\hline
\end{tabular}

Cleaning index can be defined as equation (2).

$$
\mu_{c}=\frac{\sum_{j=1}^{4} L_{c i j} \times \theta_{j}}{\sum_{j=1}^{4} L_{c a j} \times \theta_{j}}
$$

Where, $L_{c i j}$ is the ideal number of parts to be processed by the $j t h$ cleaning method, $L_{c a j}$ is the actually number of part processed by $j$ th cleaning method. $\theta_{j}$ is the cleaning score of the $j$ th cleaning method.

\subsection{Testing index $\mu_{t}$}

Testing is to determine the performance state and surface size of the used parts by means of specific detection techniques and methods. Parts inner quality detection is mainly through the nondestructive testing technology, include ultrasonic testing (UT), magnetic particle testing (MPT), eddy current testing (ET). Assuming the difficulty coefficient of UT is 0.40 , MPT is 0.60 , ET is 0.90 . The testing index can be expressed as equation (3)

$$
\mu_{t}=1-\frac{\sum_{j=1}^{n} L_{t i j} \times \delta_{j}}{\sum_{j=1}^{n} L_{t a j}}
$$

Where, $L_{t i j}$ and $L_{t a j}$ respectively represent the ideal and actual number of parts in the $j t h$ testing method, and $\delta_{j}$ is the difficulty coefficient of the $j t h$ testing method.

\section{$2.4 \quad$ Repairing index $\mu_{r}$}

Remanufacturing repairing refers to the process of reproducing used parts to recover the performance by means of advanced surface engineering and traditional machining. The common remanufacturing repairing methods are: electric arc spraying technology, plasma spraying technology, plasma cladding technology, nano composite brush plating technology and laser cladding, identified as A, B, C, D and E.

Five repairing methods are applicable to different occasions [165-171]. according to the parts repairing demand, processing requirement and repairing cost, the relative difficulty coefficient of the method is represented by comparison matrix, shown in table 2 , the method of scoring is similar to the cleaning. The repairing index is expressed as equation (4).

$$
\mu_{r}=\frac{\sum_{j=1}^{5} L_{r i j} \times \sigma_{j}}{\sum_{j=1}^{5} L_{r a j} \times \sigma_{j}}
$$


Where, $L_{r i j}$ and $L_{r a j}$ is the ideal and actual number of parts processed by the $j t h$ repairing method, $\sigma_{j}$ represents the repairing score of the $j t h$ repairing method.

Table 2 The determination of repairing score

\begin{tabular}{|c|c|c|c|c|c|c|c|c|c|}
\hline \multirow{2}{*}{$\begin{array}{l}\text { Repairing } \\
\text { method } \\
\text { (Row) }\end{array}$} & \multicolumn{5}{|c|}{ Repairing method (Column) } & \multirow{2}{*}{ Score } & \multirow{2}{*}{$\begin{array}{l}\text { Relative } \\
\text { importance } \\
/(\%)\end{array}$} & \multirow{2}{*}{$\begin{array}{l}\text { Approximate } \\
\text { repairing } \\
\text { score }\end{array}$} & \multirow{2}{*}{$\begin{array}{l}\text { Repairi } \\
\text { ng score }\end{array}$} \\
\hline & A & B & C & D & $\mathrm{E}$ & & & & \\
\hline A & 1.0 & 0.3 & 0.25 & 0.2 & 0.17 & 1.75 & 4 & 1.00 & 1 \\
\hline B & 3.0 & 1.0 & 0.3 & 0.25 & 0.20 & 4.55 & 10 & 2.50 & 3 \\
\hline $\mathrm{C}$ & 4.0 & 3.0 & 1.0 & 0.3 & 0.25 & 8.3 & 18 & 4.50 & 5 \\
\hline D & 5.0 & 4.0 & 3.0 & 1.0 & 0.5 & 13.0 & 29 & 7.25 & 7 \\
\hline $\mathrm{E}$ & 6.0 & 5.0 & 4.0 & 2.0 & 1.0 & 18.0 & 39 & 9.75 & 10 \\
\hline Total & & & & & & 45.6 & 100 & 25 & - \\
\hline
\end{tabular}

\subsection{Assembly index $\mu_{a}$}

Assembly is the process of assembling new replacement parts, repairing parts, and new modules to remanufactured products according to the remanufacturing technology requirements. Because the specified assembly way of parts, such as welding, riveting, threaded connections, the assembly process, equipment and means are fixed, therefore, assembly index can be expressed as equation (5):

$$
\mu_{a}=1
$$

\subsection{Calculation and determination of the remanufacturability index}

According to equation $(1) \sim(5)$, the comprehensive technical remanufacturability index $R$ can be obtained, expressed as equation (6), where $\omega_{i}$ is the weight of the $i t h$ index, which can be obtained by expert experience or AHP method.

$$
\begin{gathered}
R=f\left(\mu_{d}, \mu_{c} \ldots \mu_{a}\right)=\sum_{i=1}^{5} \omega_{i} \mu_{i} \\
\sum_{i=1}^{5} \omega_{i}=1
\end{gathered}
$$

Since the value range of the five technical feasibility index is $[0,1]$, the value range of corresponding remanufacturability $T$ is also between $[0,1]$, and the critical threshold value of $T$ and mechanical product remanufacturability is determined as equation (7).

$$
R=\left\{\begin{array}{cc}
{[0.9,1)} & \text { Re manufactaribility is very high } \\
{[0.8,0.9)} & \text { Re manufactaribility is high } \\
{[0.6,0.8)} & \text { Re manufactaribility is medium } \\
{[0.4,0.6)} & \text { Re manufactaribility is low } \\
{[0.2,0.4)} & \text { Re manufactaribility isvery low } \\
{[0,0.2)} & \text { No remanufactaribility }
\end{array}\right.
$$

\section{Case study}

Taking the WD615.87 engine remanufactured by Jinan Fuqiang power co., LTD (six-cylinder, water-cooled, total capacity of 9.726 liters) as an example, the original engine is used for five years, traveling 300000 kilometers.

\subsection{Remanufacturing technical feasibility index of waste engine}

Generally, the seven components that need to be considered in the process of engine remanufacturing are crankshaft, connecting rod, flywheel shell, gear room, cylinder block, cylinder cover and flywheel. The remanufacturing processes of waste engine as follows: high pressure air gun dissemble-parts high temperature washing,sand washing-magnetic particle testing-repair (plasma cladding and nano brush 
plating) - assembly.Table 3 shows the results of remanufacturing technical feasibility index and weight. Where, the weighs are determined by the company technical engineering.

Table 3 Technical feasibility index values

\begin{tabular}{lccccc}
\hline Index scores & $\mu_{\mathrm{d}}$ & $\mu_{\mathrm{c}}$ & $\mu_{\mathrm{t}}$ & $\mu_{\mathrm{r}}$ & $\mu_{\mathrm{a}}$ \\
\hline Index $\left(\mu_{\mathrm{i}}\right)$ & 0.95 & 0.74 & 0.80 & 0.88 & 0.95 \\
weight $(\omega \mathrm{i})$ & 0.15 & 0.25 & 0.18 & 0.28 & 0.14 \\
\hline
\end{tabular}

\subsection{Remanufacturability analysis and evaluation}

From Table 3 and equation (6), the remanufacturability index $T$ of the waste WD615.87 engine can be calculated as:

$$
T=f\left(\mu_{d}, \mu_{c} \ldots \mu_{a}\right)=\sum_{j=1}^{5} \omega_{i} T_{i}\left(\mu_{i}\right)=0.85
$$

By equation (7), it can be determined that the WD615.87 remanufactured engine is with good technical remanufacturability.

\section{Conclusion}

In this study, a remanufactarability evaluation method of waste mechanical product is introduced based on remanufacturing process, the calculation methods of five remanufacturing technical feasibility indexes of disassembly, cleaning, inspection, repair, and assembly are proposed, and the evaluation standard of remanufacturability is put forward, finally waste WD615.87 engine as the case study, the remanufacturing technical feasibility is analyzed and evaluated, the results show that the engine is with a good technical remanufacturability.

\section{Acknowledgement}

This research was financially supported by the 2017 Liaoning Province Natural Science Fund Guidance Project (Grant NO. 20170540080).

\section{References}

[1] B. Lebreton, Tuma A, A quantitative approach to assessing the profitability of car and truck tire remanufacturing, International Journal of Production Economic, vol.104, pp.639-652, 2006.

[2] H.C. Fang, S.K. Ong, A.Y.C. Nee, Product remanufacturability assessment and implementation based on design features, Procedia CIRP, vol. 26, pp.571-576, 2015.

[3] C.C. Li, C.B. Li, H.J. Cao, Uncertain remanufacturing process routings model for used components based on GERT network, Computer Integrated Manufacturing System, vol.18, pp.298-305, 2012.

[4] B. Bras, R. Hammond, Towards design for remanufacturing-metrics for assessing remanufacturing. Proceedings of the 1st International Workshop on Reuse, Netherlands, November 11-13, 1996. 